\title{
XXXV. On the appreciation of difference of phase of sound-waves
}

\section{L.T. More Ph.D. \& H.S. Fry Ph.D.}

To cite this article: L.T. More Ph.D. \& H.S. Fry Ph.D. (1907) XXXV. On the appreciation of difference of phase of sound-waves, Philosophical Magazine Series 6, 13:76, 452-459, DOI: $10.1080 / 14786440709463620$

To link to this article: http://dx.doi.org/10.1080/14786440709463620

曲 Published online: 16 Apr 2009.

Submit your article to this journal $\sqsubset \pi$

Џ Article views: 8

Q View related articles $\square$

Citing articles: 1 View citing articles $\square$ 
caused by any irregularity in a musical string on a violin or harp, and illustrate the impurity of tone which must follow. The curves show also that these irregularities are all taken up by the bridge, and so no doubt extend to the sound-box and the adjacent air.

Looking now over the results as a whole for the bridge's motion, it is noteworthy that the fundamental tone of the string is also revealed in the vibration of the bridge. Whereas it might have been thought that whether the string were up or down the bridge would be equally deflected inwards towards the string's centre*. But if this were the case the fundamental would be lost by the bridge, its slowest vibration being the octave. This, however, is not the case, as all experience show's and as is confirmed by the curves.

As to the general character of the vibrations of the bridge, they seem to be intermediate in type between those of the belly dealt with in the first paper and those of the air dealt with in the second.

University College, Nottingham, Dec. $21,1906$.

$\mathrm{XXXV}$. On the Appreciation of Difference of Phase of SoundWaves. By L. 'T. Mone, P'h.I'., Professor of Physies, and H. S. Fry, Ph.D., Instructor in Chemistry, The University of Cincinnati†.

$7 \mathrm{YHE}$ experiments described in this paper, in which an 1 attempt has been made to show that we can locate sounds by an appreciation of the difference of phase of the waves coming to the ears, were earried out during the winter of 1902. Certain psychologists, to whom the results were submitted, considered them to be inconclusive, and their publication was postponed until additional work could be done. But this winter Lord Rayleigh, in his Sidgwick lecture $\ddagger$, described some experiments he had performed which convinced him that we do locate sounds, partially at least, by this means. As his method differs considerably from ours, it seems best to publish these in spite of some incompleteness.

There have been several attempts made to prove that the ear is capable of detecting phase-differences of sound-waves as well as their amplitude and complexity. Apparently the attempts have not had much success, and König is perhaps the only one who maintained that one conld distinguish two

* Compare the longitudinal form of Melde's experiment, see Rayleigh's 'Theory of sound,' 2nd ed. vol. i. p. 81.

+ Communicated by the Authors.

‡ See Phil. Mag. [6] xiii. p. 214 (1907). 
complex tones similar in all respects, save the phase relations of their harmonics. His experiments with his rotating "phase-disks" have been repeated in many laboratories, and most observers agree with Helmholtz that there was no certainty in distinguishing the tones. In fact the theory of audition elaborated by Helmholtz apparently excludes the recognition of the phase of the wave by the auditory nerves. While this may be true of the sensation in a single ear, the experiments described in this paper make it probable that phase relations of sounds do influence binaural andition and enable us to locate sounds.

If we accept Helmholtz's theory of sound perception, we are, in explaining our method of locating sounds, compelled to rely on the ability of the mind to judge the intensity and complexity of the waves which enter the ears. That is; sounds coming from the right or left are louder or more complex in one ear than in the other, and by comparing the relative intensities or complexities of the two, we determine with considerable accuracy the direction of a sound.

The effect of the complexity of the wave we know little about from direct experiment and it is probably of minor importance in the general problem, as one can locate pure tones quite accurately. However, it seems natural to suppose the sound heard by the averted ear is weaker than the impression made on the other, and that the mind is capable by comparison to judge the location of the sound. But the work of Lord Rayleigh has raised very grave objections to this as an adequate method of locating sounds in all cases. We can dismiss at once the fact that one ear is a little further from the sound source than the other, because we can as readily determine the direction of sounds coming from a great distance as of those from near by. But the intensity of sounds is affected by obstacles in their path, and it is true that the head is generally more in the path of the wave to one ear than to the other. Although the head may be thus interposed, the calculations of Lord Rayleigh, 'Theory of Sound,' vol. ii. p. 442, show that it is too small an obstacle to affect appreciably the intensity of the long waves given out by grave tones. And this can be verified experimentally. If these two functions of sound-waves are inadequate, we are compelled to fall back on the only other property of a wave, its phase.

In one case, at least, we do know that the mind takes notice of the phase relations. Professor S. P. Thompson proved this by applying the receivers of two telephones, each to an ear. When the telephone disks vibrated so as to give 
waves in the same phase, the impression on the mind was quite different from that when the disks vibrated oppositely. But no experiments had then been published which showed that our power of locating sounds is largely due to this phase interpretation*.

There are three reasons which support this theory: first, experiments made on animals; second, the fact that we can locate complex tones made in the medial plane between the ears; third, the systematic experiments given below.

It is a significant fact that the ears (certainly at least the outer parts) of many animals which have need to locate sounds accurately, are placed on top of the head and close together. This position is such that the head can mako absolutely no sound shadow unless the source of the sound is directly below them. A sound coming from a considerable distance to a horse must affect each of his ears with the same intensity. Yet a horse, or any other of the animals tried, had no difficulty in locating the direction of a noise. This can be readily proved. If a person stations himself behind and somewhat to the right of an animal and then whistles, the animal invariably moves his head or ears to the right and continues the motion until pointing directly to the person, although he may be hidden. Of course the same holds true for experiments made on the left side. The results are quite certain, that such animals locate sounds accurately and yet have no means of doing this by comparing the intensity of the sounds in the two ears.

It is well known that a pure tone made directly in front of a blind-folded person cannot be distinguished from a similar tone sounded directly behind. But one has not much difficulty in distingushing complex tones under like circumstances, and especially is this true when the tones are made by the human voice. As neither a difference in intensity nor in phase exists, we were led to the belief that a complex tone produced a different sensation according to the direction the waves entered the outside ear. Certainly the shape of the outer ear is well adapted to modify the short waves of the higher harmonics, and thus to change the character of complex tones.

Lastly, we devised a set of experiments to see if the apparent direction of a simple tone could not be changed by

* The preceding remarks are, of course, no longer correct, as Lord Rayleigh, in his Sidgwick lecture of last fall and in his paper in the February issue of this journal, states his unqualified conviction that phase-differences do affect the hearing. But, as they give the views held at the time our experiments were made and in fact were the cause of their being made, we decided not to alter them. 
producing a difference in phase of the waves which entered the two ears. It was easy to produce such an effect, but an exact relation between phase-difference and direction was more difficult to obtain, although, allowing for personal variations, something has been accomplished.

A listener seated himself at the centre of a large circle marked on the floor of a room. The circle was divided by radii every $221^{\circ}$, so that $0^{\circ}$ was directly behind him and $180^{\circ}$ in front, $+90^{\circ}$ being to his right and $-90^{\circ}$ to his left. A glass funnel, $13.5 \mathrm{~cm}$. in diameter, was mounted horizontally on a table about seven feet behind him. Heavy rubber tubing, $1.2 \mathrm{~cm}$. internal diameter, connected the fumnel to a glass $\mathbf{Y}$ tube, on the other two branches of which, rubber tubing of the same size was fitted. These branch tubes ended in glass tubes bent so as to fit into the ears of the listener. Each of the branch tubes was cut in two in the middle and, by inserting pieces of glass tubing, their lengths were readily altered without the listener being at all aware of it. Fourteen different students of the college assisted, and to be sure they might not be biassed in their judgments, they remained unacquainted with the purpose of the experiment. In experimenting, a tuning-fork or the voice singing an open vowel at the same pitch was sounded at the mouth of the funnel. The listener was then asked to locate the direetion from which the sound appeared to come; this direction was compared with the angles of the circle, and the relative lengths of the branch tubes were measured.

The results, in general terms, prove that when the branch tubes were of exactly equal length, the sound seemed to come from behind, but if one were longer than the other, by say two centimetres, the direction of the sound was shifted to the right or left; when the tube to the left ear was the longer the sound came from the right, and viee versa. However the different observers might vary as to the exact angle, they were unanimous so far. Some objections to the experiments are obvious, but they can be discussed better after the results are displayed in detail.

In the following two tables of results, the capital letters stand for the different listeners. $\delta \phi$ is the difference in phase of the two vanes of sound when they reach the ears, expressed in eighths of the wave-length of the tone used. The angles give the apparent direction of the sound for various phase-differences noted by each listener. Since no mistakes were made in respect to the general directions, right. and left, it is not necessary to distinguish the angles with plus and minus signs, if we remember only that $0^{\circ}$ signifies 
456 Prof. More and Dr. Fry on the Appreciation

behind and $180^{\circ}$ in front. When the experiments were made, the order of changing the lengths of the tubes was entirely arbitrary, that is for a given observer the two tubes might be equal for the first observation, then the right tube might be lengthened a half wave-length, or the left tube be lengthened a quarter of a wave-length and so on.

TABLE I.

Pitch of fork $=512$. Wave-length $(\lambda)=64 \mathrm{~cm}$.

\begin{tabular}{|c|c|c|c|c|c|c|c|c|c|}
\hline & $\delta \phi=0$. & $\delta \phi=\frac{1}{\delta} \lambda$. & $\frac{1}{4} \lambda$. & 3. & $\frac{1}{2} \lambda$. & $\frac{5}{5} \lambda$. & ${ }_{3}^{3} \lambda$. & $\pi \lambda$ & $\lambda$. \\
\hline A. & 0 & 285 & $60 \%$ & $6 \%$ & 6 & $90^{\circ}$ & 90 & $67^{\circ} \%$ & 675 \\
\hline B & 0 & 29.5 & 45 & 67.5 & go & 675 & 90 & 67.5 & 45 \\
\hline C.. & 0 & 0 & $22 \cdot 5$ & $4 \bar{j}$ & 4.5 & 675 & 90 & 67.5 & 675 \\
\hline D . & 0 & 225 & $22 \cdot 5$ & 675 & 67.5 & 90 & 45 & 45 & 67.5 \\
\hline E & 0 & 225 & $67 \cdot 5$ & 675 & 45 & 675 & 45 & 675 & 67.5 \\
\hline F & 0 & 2 & 45 & 675 & 90 & 675 & 675 & 67.5 & 90 \\
\hline a. & 0 & $22: 5$ & 225 & $4 \bar{\jmath}$ & 675 & 90 & 45 & 225 & 0 \\
\hline H. & 0 & 5 & $4 \overline{3}$ & 65 & 675 & 225 & 90 & 80 & 30 \\
\hline I .. & 0 & $22=5$ & 45 & 67.5 & 90 & 675 & 90 & 90 & 112.5 \\
\hline IK ... & 0 & 225 & 67.5 & 90 & $67 \cdot \tilde{J}$ & 45 & 67.5 & 45 & 90 \\
\hline
\end{tabular}

Table II.

Pitch of fork $=320$. Wave-length $(\lambda)=104 \mathrm{~cm}$.

\begin{tabular}{|c|c|c|c|c|c|c|c|c|c|}
\hline & $\delta \phi=0$. & $\delta \phi=\frac{T}{\gamma} \lambda$. & ${ }_{4}^{1} \lambda \lambda$. & ${ }_{5}^{8} \lambda$. & $\frac{i}{2} \lambda$. & $\pi \lambda$. & ${ }_{4}^{3} \lambda$. & $\frac{7}{8} \lambda$. & $\lambda$. \\
\hline L ... & 0 & $2: 5$ & 45 & $4 \stackrel{\circ}{15}$ & 90 & $90^{\circ}$ & 90 & 90 & $90^{\circ}$ \\
\hline$M \ldots$ & 0 & 225 & 45 & 675 & 675 & 67.5 & 90 & 90 & 90 \\
\hline$N \ldots$ & $\hat{0}$ & 22.5 & 90 & 90 & 90 & 90 & 90 & $67 \cdot 5$ & 90 \\
\hline o... & ; & 225 & 675 & 45 & 225 & 675 & 90 & o0 & 90 \\
\hline$c \ldots$ & $?$ & 225 & 45 & $6 \% 5$ & 90 & 90 & 90 & 90 & 90 \\
\hline F ... & 0 & $4 \overline{5}$ & 67.5 & 67.5 & 675 & 675 & $67 \cdot 5$ & 675 & 90 \\
\hline I. ... & 0 & 225 & $4 ;$ & $67 \cdot 5$ & 675 & 90 & 90 & 90 & 90 \\
\hline
\end{tabular}

In spite of a certain indefiniteness in the results and considerable variations in the exact direction for any given phase-difference, the observations of these fourteen students, who, without an exception, correctly noted changes of direction, go to show that the hearing is affected by phase variation in the waves reaching the ears. Examining the data mure carefully, we can divide them into two parts. Up to a 
certain increase in phase-difference, the change of direction increases regularly and uniformly. But when the phasedifference becomes greater than 3 , for $\lambda=64 \mathrm{~cm}$., and $\frac{1}{4} \lambda$ for $\lambda=104 \mathrm{~cm}$., decision is more difficult, the apparent direction remaining unchanged to some and to otbers the angle is diminished. This may be shown by the following summary :-

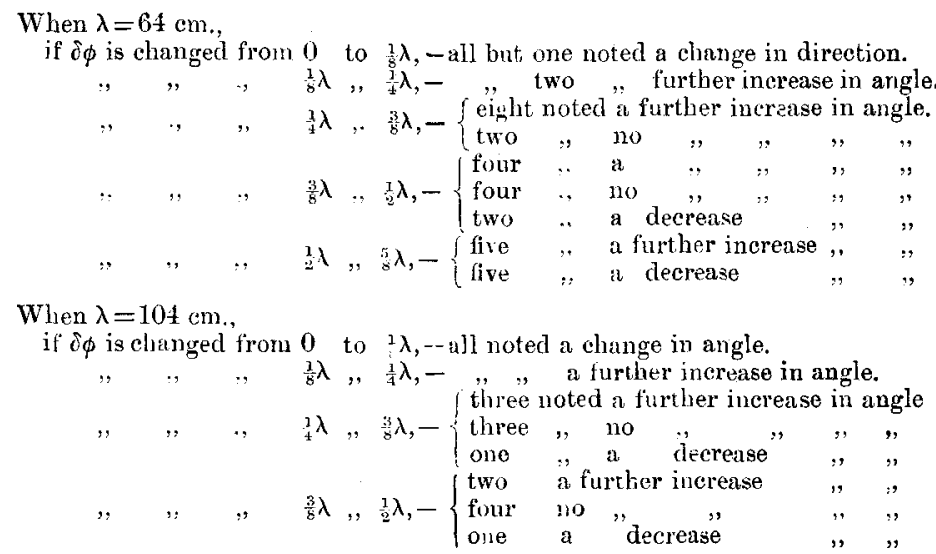

We may then say, for the wave-lengths used, a phasedifference up to about a quarter of a wave-length determines partially or wholly the apparent direction of a sound. From the properties of summation wave forms, we should expect a difference of phase of a quarter wave-length to be the greatest which would alter the form of the wave made by this addition. The agreement between our observations and this property of waves cannot, however, be said to be established except for these wave-lengths, and experiments to test this point should be made with sounds presenting great differences in pitch.

The experiments were continued up to a phase-difference of a whole wave-length, and roughly for greater differences, but the latter were not recorded as the angle continued, with most observers, to be about $90^{\circ}$.

Analytically, of course, the complex waves are identical when the phase-differences of the component waves are zero or a whole wave-length; and to explain the fact that most observers noticed a difference in direction for these two cases, we are led to believe that when one tube is so much longer than the other, the intensity of the sound in the longer tube then becomes appreciably less than in the other. This supposition is supported by the fact that the majority found 


\section{Appreciation of Difference of Phase of Sound-Waves.}

a maximum change in direction before the phase-difference of a wave-length was reached for the fork of shorter wavelength, but did not find such a maximum for the graver tone. And also we should remember that in the problem of locating sounds of these pitches in the free air, such large differences of phase would not occur. For example, the average distance between the ears of a man is about 15.5 centimetres, and that is less than a quarter of these wavelengths.

If it be granted that we locate sounds entirely by their phase relations up to a phase-difference determined by the distance between the ears, we still find this insutficient as an explanation for locating sounds which come from the extreme right or left. For these large angles, difference in intensity may be our principal aid, especially as the head is best fitted then to cast a sound shadow. It is also a matter of observation that it is not so easy to locate either very grave tones or very shrill ones, and this may possibly be so because the difference in path to produce a phase-difference of a quarter of a wave-length is either mach greater or much less than the width of the head.

During the experiments, attention was paid to the question whether difference in phase produced a change in the intensity of the combined tones. Analytically two sounds interfere when the phase-difference is a half wave-length. But the mind evidently does not combine the separate sensations in the ears in so simple a manner, as not one of the observers could detect any alteration in the loudness of the sounds when the relative phases were changed. Neither were they heard as two tones, nor was the sound louder in one ear than in the other. The effect was as if the fork had been sounded in the free air at different points on the circumference of a circle.

These experiments are perhaps open to the objection that, to produce a difference in phase, one branch tube must be made longer than the other. And so a doubt may arise whether the intensity of the sound going through the longer tube is not diminished more than that through the other tube. But the observations show that this objection is not valid. If it were a question of change of intensity, the change in direction would increase continually and not reach an angle where further increase in length of the tube produces either a doubtful increase in angle or even at times a decrease. We tested the least change in the relative lengths of the tubes which would shift the angle appreciably and found, for some listeners, two centimetres to be sufficient. The 
Convective Equilibrium of a Spherical Mass of Gas. 459

diminution in intensity for a length of two centimetres of large tubing would be quite unnoticeable. Also it should be remembered that, to explain the location of a sound by differences in intensity, the idea of a sound shadow produced by the head was introduced, as it was evident that the difference in length of path of the sound to the two ears was not sufficient a cause.

The writers of this paper are not well enough acquainted with physiology to offer any explanation of the way the mind takes cognisance of phase-differences of tones. The theory of the mechanism of bearing advanced by Helmholtz seems entirely unsuitable, for a simple rod-like filament of the auditory nerve, vibrating sympathetically to waves of sound, apparently has no apparatus for recognizing phase-differences. So far as we know there has been but little adverse criticism of Helmholtz's work on the ear except in one instance. Dr. Howard Ayers, after a careful and laborious examination and measurement, maintains that his theory of audition is incorrect. Unfortunately the work of Dr. Ayers is not at hand, so a comparison of his theory with our experiments is not possible at present.

Before the laws of this effect can be stated completely a large number of experiments must be made, and it is hoped that those planned to be done this year will prove adequate.

Cambridge, England, January $190 \%$.

XXXVI. On Convective Equilibrium of a Spherical Mass of Gas subject only to the Mutual Gravitation of its parts. By J. PrescotT, Lecturer in Mathematics at the Manchester Seloool of Technology*.

PEFORE attacking the question from a mathematical B standpoint, let us get some idea from physical considerations, how the radius of the sphere of zero density depends on the mass of the gas.

Suppose that, when a mass of gas acted on by no forces except its own gravitation has taken up its equilibrium state, it were possible suddenly to double its density at every point by placing in every portion of space an exactly equal and similar mass of gas to that which already occupies the space. The pressure would be doubled everywhere, and therefore the resultant of the pressures acting on the gas enclosed within any given space would be doubled. But the mass within the space would be doubled, and the attracting mass

* Communicated by the Author. 